\title{
Arbitrarily large steady-state bosonic squeezing via dissipation
}

\author{
Andreas Kronwald, ${ }^{1, *}$ Florian Marquardt,,${ }^{1,2}$ and Aashish A. Clerk ${ }^{3}$ \\ ${ }^{1}$ Friedrich-Alexander-Universität Erlangen-Nürnberg, Staudtstrasse 7, D-91058 Erlangen, Germany \\ ${ }^{2}$ Max Planck Institute for the Science of Light, Günther-Scharowsky-Straße 1/Bau 24, D-91058 Erlangen, Germany \\ ${ }^{3}$ Department of Physics, McGill University, Montreal, Quebec, Canada H3A 2 T8
}

(Received 23 July 2013; revised manuscript received 11 November 2013; published 19 December 2013)

\begin{abstract}
We discuss how large amounts of steady-state quantum squeezing (beyond $3 \mathrm{~dB}$ ) of a mechanical resonator can be obtained by driving an optomechanical cavity with two control lasers with differing amplitudes. The scheme does not rely on any explicit measurement or feedback, nor does it simply involve a modulation of an optical spring constant. Instead, it uses a dissipative mechanism with the driven cavity acting as an engineered reservoir. It can equivalently be viewed as a coherent feedback process, obtained by minimally perturbing the quantum nondemolition measurement of a single mechanical quadrature. This shows that in general the concepts of coherent feedback schemes and reservoir engineering are closely related. We analyze how to optimize the scheme, how the squeezing scales with system parameters, and how it may be directly detected from the cavity output. Our scheme is extremely general, and could also be implemented with, e.g., superconducting circuits.
\end{abstract}

DOI: 10.1103/PhysRevA.88.063833

PACS number(s): 42.50.Pq, 42.50.Nn, 07.10.Cm, 85.85.+j

\section{INTRODUCTION}

Among the simplest nonclassical states of a harmonic oscillator are quantum squeezed states, where the uncertainty of a single motional quadrature is suppressed below the zero-point level [1]. Such states are of interest for a variety of applications in ultrasensitive force detection [3]; they are also a general resource for continuous variable quantum-information processing [4]. It has long been known that coherent parametric driving can be used to generate squeezing of a bosonic mode; for a mechanical resonator, this simply amounts to modulating the spring constant at twice the mechanical resonance frequency [5]. Such a simple parametric interaction can yield at best steady-state squeezing by a factor of $1 / 2$ below the zero-point level (the so-called $3 \mathrm{~dB}$ limit) [6]; if one further increases the interaction strength, the system becomes unstable and starts to self-oscillate.

The rapid progress in quantum optomechanics [7-10], where a driven electromagnetic cavity is used to detect and control a mechanical resonator, has led to a renewed interest in the generation of squeezing. One could simply use radiation pressure forces to define an oscillating spring constant [11-15], though this cannot surpass the usual $3 \mathrm{~dB}$ limit on stationary squeezing. One can do better by combining continuous quantum measurements and feedback, either by making a quantum nondemolition (QND) measurement of a single motional quadrature [3,16-18], or by combining detuned parametric driving with position measurement $[19,20]$. While such schemes can generate quantum squeezing well past the $3 \mathrm{~dB}$ limit, they are difficult to implement, as they require near-ideal measurements and feedback. The $3 \mathrm{~dB}$ limit could also be surpassed by continuously injecting squeezed light directly into the cavity [21], but this is also difficult as one needs to start with a source of highly squeezed light. We note

\footnotetext{
*andreas.kronwald@physik.uni-erlangen.de
}

that a mechanical resonator could also be squeezed via a pulsed optomechanical scheme [22].

In this work, we discuss a remarkably simple scheme for generating steady-state mechanical squeezing well beyond the $3 \mathrm{~dB}$ level. We use a two-tone driving of an optomechanical cavity, without any explicit measurement or feedback [cf. Fig. 1(a)]. As described in Refs. [3,16,18], if one drives the cavity with equal amplitude at both $\omega_{\text {cav }} \pm \Omega$ (where $\omega_{\text {cav }}$ is the cavity frequency and $\Omega$ is the mechanical frequency), then the cavity only couples to a single mechanical quadrature $X_{1}$, allowing for a QND measurement and preventing any backaction disturbance of $X_{1}$. In contrast, we consider a situation in which the two drive tones have different amplitudes. One thus no longer has a QND situation, and there will be a backaction disturbance of $X_{1}$. However, this disturbance acts to suppress (a)

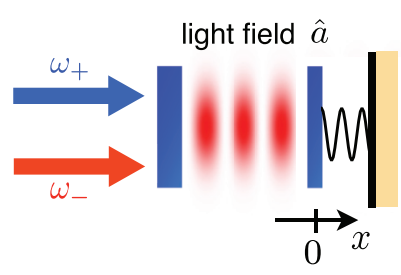

(b)

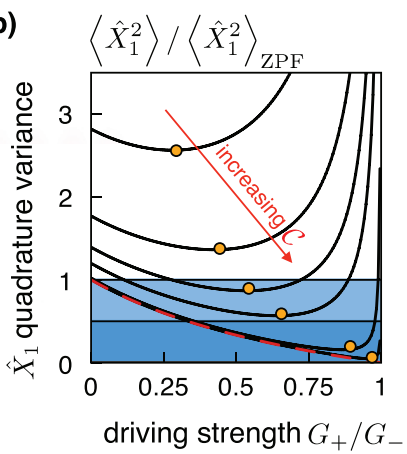

FIG. 1. (Color online) (a) An optomechanical cavity is driven on the red and blue mechanical sideband with different laser amplitudes. This leads to steady-state mechanical squeezing beyond $3 \mathrm{~dB}$. (b) Steady-state quadrature fluctuations $\left\langle\hat{X}_{1}^{2}\right\rangle$ [in units of the zero-point fluctuations (ZPF)] as a function of the blue laser driving strength $G_{+}$for different cooperativities $\mathcal{C}=4 G_{-}^{2} /\left(\kappa \Gamma_{M}\right)$. The dark blue region indicates squeezing beyond $3 \mathrm{~dB}$. The red dashed line is the variance of a squeezed vacuum state with squeeze parameter $r=\arctan G_{-} / G_{+}$. An optimal choice of $G_{+} / G_{-}$exists (orange circles) maximizing the amount of squeezing for each $\mathcal{C}$. (Parameters: $\Gamma_{M} / \kappa=10^{-4}, n_{\mathrm{th}}=10, \mathcal{C}=10,25,50,10^{2}, 10^{3}, 10^{4}$.) 
the fluctuations of $X_{1}$, to a level even below the zero-point level. Our scheme thus realizes a coherent feedback operation, where the driven cavity both measures the $X_{1}$ quadrature and autonomously applies the corresponding feedback operation necessary to "cool" $X_{1}$ (cf. Appendix A and Refs. [23-25] for other examples of coherent feedback in optomechanics). This, hence, suggests the general recipe to construct a coherent feedback scheme by perturbing a QND measurement setup minimally. Equivalently, one can think of the scheme as an example of reservoir engineering [26]: the driven cavity acts effectively as a bath whose force noise is squeezed. This suggests that in general the concepts of coherent feedback and reservoir engineering are closely related.

In what follows, we provide a thorough analysis of the optimal steady-state squeezing generated by our scheme, showing that the squeezing is a sensitive function of the ratio of the cavity drive amplitudes. We also show that for realistic parameters, one can obtain mechanical squeezing well beyond the usual $3 \mathrm{~dB}$ limit associated with a coherent parametric driving. While we focus here on optomechanics, our scheme could also be realized in other implementations of parametrically coupled bosonic modes, e.g., superconducting circuits [27,28]. Note that related dissipative mechanisms can be utilized to prepare the motion of a trapped ion in a squeezed state [29], to squeeze a mechanical resonator which is coupled to a two-level system [30], and to produce spin squeezing of atoms in a cavity [31,32]. Unlike those works, our analysis does not rely on describing the engineered reservoir (the driven cavity) via a simple Lindblad master equation; in fact, we explicitly discuss corrections to such an approximation, which we show to become significant for current experiments. We also note that reservoir engineering approaches to optomechanics have been previously considered for generating entanglement (two-mode squeezing) [33,34], as well as coherence in arrays [35].

\section{MODEL}

We consider a standard optomechanical system, where a single cavity mode couples to a mechanical resonator via radiation pressure; cf. Fig. 1(a). It is described by the optomechanical Hamiltonian [36]

$$
\hat{H}=\hbar \omega_{\mathrm{cav}} \hat{a}^{\dagger} \hat{a}+\hbar \Omega \hat{b}^{\dagger} \hat{b}-\hbar g_{0} \hat{a}^{\dagger} \hat{a}\left(\hat{b}^{\dagger}+\hat{b}\right)+\hat{H}_{\mathrm{dr}},
$$

where the two-tone laser driving Hamiltonian reads

$$
\hat{H}_{\mathrm{dr}}=\hbar\left(\alpha_{+} e^{-i \omega_{+} t}+\alpha_{-} e^{-i \omega_{-} t}\right) \hat{a}^{\dagger}+\text { H.c. }
$$

$\hat{a}(\hat{b})$ is the photon (phonon) annihilation operator, and $g_{0}$ is the optomechanical coupling. $\omega_{ \pm}$and $\alpha_{ \pm}$are the frequency and amplitude of the two lasers, respectively. We apply the displacement transformation $\hat{a}=\bar{a}_{+} e^{-i \omega_{+} t}+\bar{a}_{-} e^{-i \omega_{-} t}+$ $\hat{d}$ to (1) and go into an interaction picture with respect to the free cavity and mechanical resonator Hamiltonian. Here, $\bar{a}_{ \pm}$is the coherent light field amplitude due to the two lasers. If $\Omega$ is strongly temperature-dependent, oscillations in the average cavity intensity can yield spurious parametric instabilities [37]; these could be suppressed by adding an appropriate third drive tone without strongly degrading the generation of squeezing [38] (cf. Appendix G).
We next take the two lasers to drive the mechanical sidebands of a common mean frequency $\bar{\omega}$, i.e., $\omega_{ \pm}=\bar{\omega} \pm \Omega$, and assume $\left|\bar{a}_{ \pm}\right| \gg 1$. For $\bar{\omega}$ far detuned from $\omega_{\text {cav }}$, one can eliminate the cavity to obtain an "optical spring" which is modulated at $2 \Omega$ [14,15]; Ref. [11] obtains an analogous effect by weakly amplitude-modulating a single strong drive at $\omega_{\text {cav }}-\Omega$. In contrast, we take $\bar{\omega}=\omega_{\text {cav }}$ as well as $\bar{a}_{+} \neq \bar{a}_{-}$ [in contrast to backaction-evasion (BAE) schemes [3,16,18]]. Applying a standard linearization to Eq. (1), we find that the linearized Hamiltonian in our interaction picture is

$$
\begin{aligned}
\hat{H}= & -\hbar \hat{d}^{\dagger}\left(G_{+} \hat{b}^{\dagger}+G_{-} \hat{b}\right)+\text { H.c. } \\
& -\hbar \hat{d}^{\dagger}\left(G_{+} \hat{b} e^{-2 i \Omega t}+G_{-} \hat{b}^{\dagger} e^{2 i \Omega t}\right)+\text { H.c. }
\end{aligned}
$$

Here, $G_{ \pm}=g_{0} \bar{a}_{ \pm}$are the enhanced optomechanical coupling rates; without loss of generality, we assume $G_{+}, G_{-}>0$. The quantum Langevin equations describing the dissipative dynamics read [39]

$$
\dot{\hat{d}}=\frac{i}{\hbar}[\hat{H}, \hat{d}]-\frac{\kappa}{2} \hat{d}+\sqrt{\kappa} \hat{d}_{\text {in }} .
$$

A similar equation holds for $\hat{b}$, where the cavity decay rate $\kappa$ is replaced by the mechanical decay rate $\Gamma_{M}$. The nonzero input noise correlators read $\left\langle\hat{d}_{\text {in }}(t) \hat{d}_{\text {in }}^{\dagger}\left(t^{\prime}\right)\right\rangle=\delta(t-$ $\left.t^{\prime}\right), \quad\left\langle\hat{b}_{\text {in }}(t) \hat{b}_{\text {in }}^{\dagger}\left(t^{\prime}\right)\right\rangle=\left(n_{\text {th }}+1\right) \delta\left(t-t^{\prime}\right)$, and $\left\langle\hat{b}_{\text {in }}^{\dagger}(t) \hat{b}_{\text {in }}\left(t^{\prime}\right)\right\rangle=$ $n_{\text {th }} \delta\left(t-t^{\prime}\right)$, where $n_{\text {th }}$ is the thermal occupancy of the mechanical bath.

\section{SQUEEZING GENERATION}

We now present two intuitive ways of understanding the generation of steady-state squeezing in our scheme. For physical transparency, we focus on the good cavity limit $\kappa \ll \Omega$, and thus ignore counterrotating terms in Eq. (2) until the last section.

Note first that if $G_{+}=G_{-}$(i.e., equal drive amplitudes), the cavity only couples to the mechanical quadrature $\hat{X}_{1}=$ $\left(\hat{b}^{\dagger}+\hat{b}\right) / \sqrt{2}$; cf. Eq. (2). As discussed earlier, this allows a QND measurement of $\hat{X}_{1}[16,18]$. If $G_{+} \neq G_{-}$, the cavity still couples to a single mechanical operator, a Bogoliubov-mode annihilation operator

$$
\hat{\beta}=\hat{b} \cosh r+\hat{b}^{\dagger} \sinh r
$$

where the squeezing parameter $r$ is defined via $\tanh r=$ $G_{+} / G_{-}$. We also assume $G_{+}<G_{-}$, which ensures stability. The Hamiltonian (2) becomes

$$
\hat{H}=-\mathcal{G} \hat{d}^{\dagger} \hat{\beta}+\text { H.c., }
$$

where the coupling $\mathcal{G}=\sqrt{G_{-}^{2}-G_{+}^{2}}$. This is a beam-splitter Hamiltonian well known from optomechanical sideband cooling $[40,41]$. However, instead of allowing the cavity to cool the mechanical mode, it can now cool the mode $\hat{\beta}$. As the vacuum of $\hat{\beta}$ is the squeezed state $\hat{S}(r)|0\rangle$ (where $\left.\hat{S}(r)=\exp \left[r\left(\hat{b} \hat{b}+\hat{b}^{\dagger} \hat{b}^{\dagger}\right) / 2\right]\right)[5]$, this cooling directly yields steady-state squeezing. In general,

$$
2\left\langle\hat{X}_{1}^{2}\right\rangle=e^{-2 r}\left[1+2\left\langle\hat{\beta}^{\dagger} \hat{\beta}\right\rangle+\langle\hat{\beta} \hat{\beta}\rangle+\left\langle\hat{\beta}^{\dagger} \hat{\beta}^{\dagger}\right\rangle\right] .
$$

If $\hat{\beta}$ is in its ground state, $2\left\langle\hat{X}_{1}^{2}\right\rangle=e^{-2 r}$. Thus, the cavity acts as an engineered reservoir that can cool the mechanical resonator into a squeezed state. We note that related 
entanglement-via-dissipation schemes [31,33,34] are based on cooling a delocalized Bogoliubov mode. In contrast, we study a localized mode which directly leads to a (single-mode) squeezed mechanical steady state.

As mentioned, one can also interpret the squeezing generation without invoking a Bogoliubov mode, but rather as a coherent feedback operation where the cavity both measures and perturbs $\hat{X}_{1}$. In the simplest large- $\kappa$ limit, the feedback causes both $\hat{X}_{1}$ and $\hat{X}_{2}=i\left(\hat{b}^{\dagger}-\hat{b}\right) / \sqrt{2}$ to be damped at a rate $\Gamma_{\text {opt }}=4 \mathcal{G}^{2} / \kappa$, but adds negligible fluctuations to $\hat{X}_{1}$ (smaller than the zero-point fluctuations that one would associate with $\Gamma_{\text {opt }}$; cf. Appendix A). Thus, despite being driven with classical light, the cavity acts as a squeezed reservoir leading to mechanical squeezing.

\section{SQUEEZING VERSUS DRIVING STRENGTHS}

We solve the quantum Langevin equations (first in the rotating-wave approximation) and consider the steady-state mechanical squeezing as a function of $G_{+} / G_{-}$, cf. Fig. 1(b), holding constant both the ratio of damping rates $\Gamma_{M} / \kappa$ and the red-laser amplitude [parametrized via the cooperativity $\left.\mathcal{C}=4 G_{-}^{2} /\left(\kappa \Gamma_{M}\right)\right]$. Without the blue-detuned laser, i.e., $G_{+}=$ 0 , we have standard optomechanical sideband cooling: both quadrature variances are reduced as compared to the thermal case $[40,41]$. Turning on $G_{+}$, the quadrature variance $\left\langle\hat{X}_{1}^{2}\right\rangle$ first decreases with increasing $G_{+} / G_{-}$. In general, $\left\langle\hat{X}_{1}^{2}\right\rangle$ exhibits a minimum as a function of $G_{+} / G_{-}$which becomes sharper with increasing cooperativity $\mathcal{C}$. For large $\mathcal{C}$, the minimum variance is well below $1 / 2$ the zero-point value, i.e., the $3 \mathrm{~dB}$ limit. This minimum results from the competition of two opposing tendencies. On one hand, increasing $G_{+} / G_{-}$ increases the squeezing parameter $r$ and thus the squeezing associated with the vacuum of $\beta$. On the other hand, increasing $G_{+} / G_{-}$reduces $\mathcal{G}$, and hence suppresses the ability of the cavity to $\operatorname{cool} \beta$. The optimum squeezing is thus a tradeoff between these tendencies.

\section{OPTIMAL SQUEEZING}

Consider a fixed red-laser amplitude (i.e., $G_{-}$) large enough that the cooperativity $\mathcal{C} \gg 1$. The value of $G_{+}$which maximizes squeezing is then

$$
\left.\frac{G_{+}}{G_{-}}\right|_{\text {optimal }} \approx 1-\sqrt{\frac{1+2 n_{\mathrm{th}}}{\mathcal{C}}}, \text { i.e., } e^{-2 r} \approx \frac{1}{2} \sqrt{\frac{1+2 n_{\mathrm{th}}}{\mathcal{C}}} .
$$

The corresponding minimum value of $\left\langle\hat{X}_{1}^{2}\right\rangle$ is

$$
2\left\langle\hat{X}_{1}^{2}\right\rangle \approx \frac{\Gamma_{M}}{\kappa}\left(1+2 n_{\mathrm{th}}\right)+\sqrt{\frac{1+2 n_{\mathrm{th}}}{\mathcal{C}}} ;
$$

cf. Fig. 2(a). We see that even for moderate values of $\mathcal{C}$ and nonzero $n_{\text {th }}$, quantum squeezing beyond $3 \mathrm{~dB}$ is achieved; cf. Fig. 2(a). As $\mathcal{C}$ is increased further, the amount of squeezing saturates to a level set by the ratio of the mechanical heating rate to $\kappa$. Note that if one attempts to describe the effect of the cavity on the mechanical resonator via an effective Lindblad master equation, one misses this saturation; cf. Fig. 2(a) (see Appendix D). An analogous Lindblad approach was recently analyzed in the context of spin squeezing in Ref. [32].
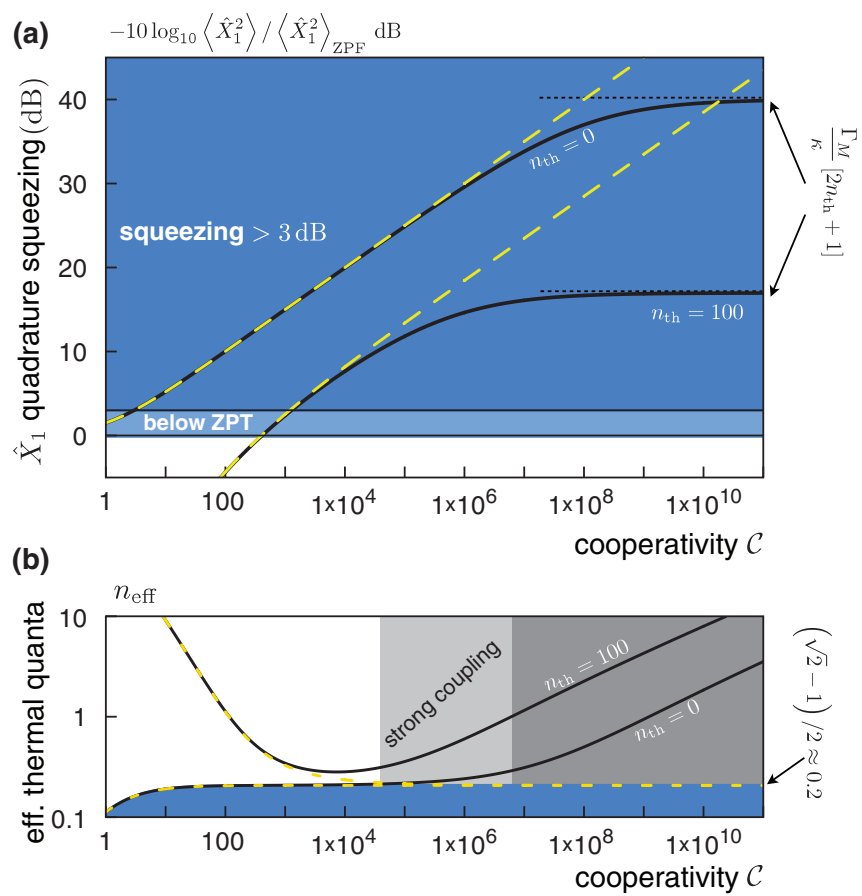

FIG. 2. (Color online) Maximized steady-state squeezing and state purity. (a) Squeezing for fixed $\Gamma_{M} / \kappa$ and optimized driving strength $G_{+}$[cf. (5)], as a function of $G_{-}$(parametrized by the cooperativity $\mathcal{C}$ ). Squeezing beyond $3 \mathrm{~dB}$ is apparent even for moderate $\mathcal{C}$. Black lines represent the full theory. These curves are well described by (6) for $\mathcal{C} \gtrsim 100$. Yellow dashed lines show the prediction of a Lindblad master equation [LME, Eq. (D1)]. (b) Effective thermal occupancy $n_{\text {eff }}$ in the mechanical steady state, for optimized parameters, as a function of $\mathcal{C}$. The colors are the same as in (a). The full theory and the LME differ drastically in the strong-coupling regime where $\mathcal{G} \gtrsim \kappa$ (gray shaded region, beginning first for $\left.n_{\mathrm{th}}=100\right)$. (Parameters: $\Gamma_{M} / \kappa=10^{-4}, n_{\mathrm{th}}=0$, and $n_{\mathrm{th}}=100$.)

\section{STATE PURITY}

It follows from Eqs. (4)-(6) that the maximal squeezing of our scheme (at fixed cooperativity $\mathcal{C}$ ) corresponds to $\left\langle\hat{\beta}^{\dagger} \hat{\beta}\right\rangle>0$. The steady state is thus a squeezed thermal state. To quantify the purity of this state, we define an effective thermal occupancy from the determinant of the mechanical covariance matrix, i.e.,

$$
\left(1+2 n_{\mathrm{eff}}\right)^{2}=4\left\langle\hat{X}_{1}^{2}\right\rangle\left\langle\hat{X}_{2}^{2}\right\rangle-4\left\langle\left\{\hat{X}_{1}, \hat{X}_{2}\right\}\right\rangle^{2} .
$$

The mechanical state is a pure squeezed vacuum state if $n_{\text {eff }}=0$, while the mixedness of the state increases with $n_{\text {eff }}$. As shown in Fig. 2(b), for moderately strong $\mathcal{C}$, one can both achieve squeezing beyond $3 \mathrm{~dB}$ and a low-entropy state, with $n_{\text {eff }} \sim(\sqrt{2}-1) / 2 \approx 0.2$ (independent of all parameters; see Appendix B). This again is in marked contrast to coherent parametric driving, where the maximal squeezing of $3 \mathrm{~dB}$ is associated with a diverging $n_{\text {eff }}$. This is also in contrast to the squeezing generated by a BAE measurement and feedback, where strong squeezing is also associated with $n_{\text {eff }} \gg 1$ (cf. [18] and Appendix C).

As we increase $\mathcal{C}$ further, we enter the strong-coupling regime where $\mathcal{G} \gtrsim \kappa, \Gamma_{M}$ and the cavity and the Bogoliubov 
mode hybridize. The squeezing saturates in this regime [cf. Eq. (6)], whereas $n_{\text {eff }}$ increases without bound. For optimized couplings, we find $n_{\text {eff }}^{2} \sim\left(\Gamma_{M} / 2 \kappa\right) \sqrt{\left(1+2 n_{\text {th }}\right) \mathcal{C}}$ in the large- $\mathcal{C}$ limit. Thus, while one can get enhanced squeezing in the strong-coupling regime, it comes at the price of strongly reducing the purity of the squeezed state. It is also worth emphasizing that, as shown in Fig. 2(b), the Lindblad master equation approximation fails to describe accurately both the quadrature squeezing and $n_{\text {eff }}$ at strong coupling; this is not surprising as the Lindblad approach cannot describe the hybridization physics important in this regime; cf. Appendix D.

\section{SQUEEZING DETECTION}

Squeezing of $X_{1}$ can be detected by making a singlequadrature backaction-evading measurement. One generates the squeezed state as described above by having $G_{+}<G_{-}$. To then measure the squeezing, one simply increases $G_{+}$ so that $G_{+}=G_{-}$, thus allowing a QND measurement of $X_{1}[16,18,38]$. The measurement must be fast compared to the rate at which the mechanical dissipation rethermalizes $X_{1}$, leading to the condition $\mathcal{C} \gtrsim 2 n_{\text {th }}+1$ [18].

A simpler method for verifying squeezing is to keep $G_{+}<$ $G_{-}$and use the cavity output spectrum to extract the occupancy of the $\hat{\beta}$ mode: photons in the output are a measure of how "hot" this mode is. This is analogous to how mechanical temperature is obtained in sideband cooling setups [40,41]. The output spectrum is given by

$$
S[\omega]=\int d t e^{i \omega t}\left\langle\delta \hat{a}_{\text {out }}^{\dagger}(t) \delta \hat{a}_{\text {out }}(0)\right\rangle .
$$

Here, $\delta \hat{a}_{\text {out }}=\hat{a}_{\text {out }}-\left\langle\hat{a}_{\text {out }}\right\rangle$ and $\hat{a}_{\text {out }}+\hat{a}_{\text {in }}=\sqrt{\kappa} \hat{a}$ [39], where we have assumed an ideal, single-sided cavity. We find in the good cavity limit (cf. Appendix E)

$$
\int d \omega S[\omega]=8 \pi \kappa \frac{\mathcal{G}^{2}}{4 \mathcal{G}^{2}+\kappa\left(\kappa+\Gamma_{M}\right)}\left\langle\hat{\beta}^{\dagger} \hat{\beta}\right\rangle .
$$

Thus, knowing the red and blue driving strength $G_{ \pm}$as well as the cavity decay rate $\kappa \gg \Gamma_{M}$ is sufficient to measure $\left\langle\hat{\beta}^{\dagger} \hat{\beta}\right\rangle$. The knowledge of $\left\langle\hat{\beta}^{\dagger} \hat{\beta}\right\rangle$ is enough to find a rigorous upper bound of the squeezing, since

$$
\left\langle\hat{X}_{1}^{2}\right\rangle \leqslant e^{-2 r}\left[1+2\left\langle\hat{\beta}^{\dagger} \hat{\beta}\right\rangle\right]
$$

(see Appendix E). For large $\mathcal{C}$, this upper bound coincides with the actual value of $\left\langle\hat{X}_{1}^{2}\right\rangle$ (up to corrections $\sim 1 / \sqrt{\mathcal{C}}$ ).

\section{EFFECTS OF COUNTERROTATING TERMS}

We now turn to the effects of the counterrotating terms in Eq. (2) which can play a role when one deviates from the extreme good cavity limit $\kappa \ll \Omega$. These additional terms cause the cavity to nonresonantly heat the Bogoliubov mode $\hat{\beta}$; as a result, the coupling-optimized quadrature squeezing becomes a nonmonotonic function of the cooperativity $\mathcal{C}$. This is shown in Fig. 3, where we have solved the full quantum Langevin equations, and we took parameters from a recent experiment in microwave cavity optomechanics [42]. Steady-state squeezing beyond $3 \mathrm{~dB}$ still exists even for only moderately resolved sidebands $(\Omega / \kappa \sim 5)$. To estimate the onset of the nonresonant heating, we can calculate the leading

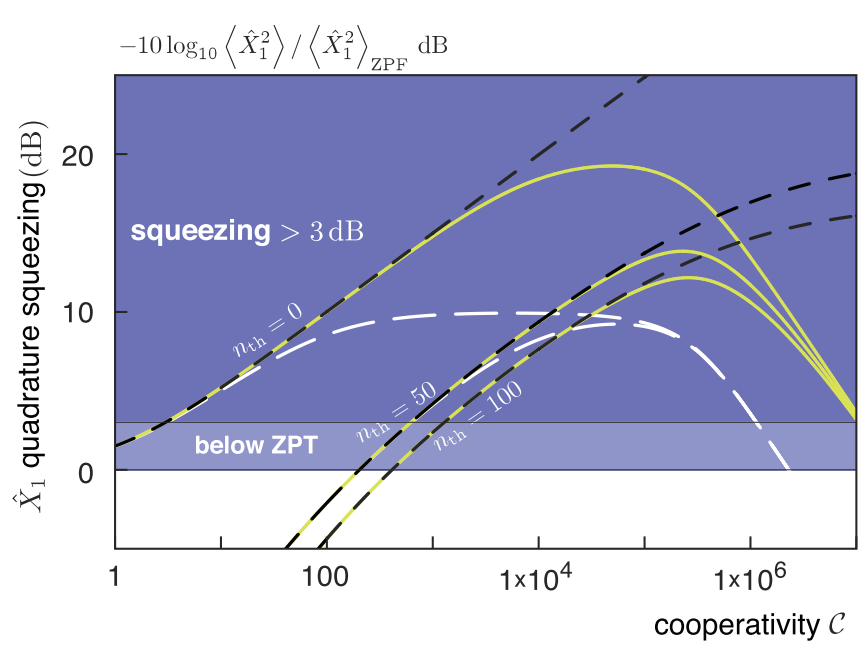

FIG. 3. (Color online) Squeezing vs $\mathcal{C}$ for optimized driving strengths, using realistic experimental parameters [42], and the full theory. Yellow and white dashed lines show the theory including effects due to a nonzero sideband parameter [i.e., no rotating-wave approximation, in contrast to Fig. 2(a)]. The black dashed line shows the expectation for $\kappa / \Omega=0$. Thus, squeezing beyond $3 \mathrm{~dB}$ is expected for state-of-the-art experiments. (Parameters: yellow lines: $\Gamma_{M} / \kappa=10^{-4}, \kappa / \Omega=1 / 50$; white dashed lines: $\Gamma_{M} / \kappa=10^{-5}$, $\kappa / \Omega=1 / 5$.)

$O\left[(\kappa / \Omega)^{2}\right]$ correction to $\left\langle\hat{X}_{1}^{2}\right\rangle$. Insisting it be much smaller than the smallest variance possible in the extreme good cavity limit (and taking $\mathcal{C} \gg 1$ ) leads to the following condition on the cooperativity:

$$
\mathcal{C}^{3 / 2} \ll \sqrt{1+2 n_{\mathrm{th}}} \frac{\kappa}{\Gamma_{M}}\left(\frac{\Omega}{\kappa}\right)^{2} .
$$

This condition also ensures that the previous results for the optimized coupling strengths remain valid. Further discussion of bad cavity effects (as well as parameters relevant to recent optical-frequency optomechanics experiments) are presented in Appendix F.

\section{CONCLUSION}

We have shown that large steady-state squeezing of a mechanical resonator can be achieved by driving an optomechanical cavity at both the red and blue mechanical sideband, with different amplitudes. For realistic parameters, steady-state quantum squeezing well beyond the $3 \mathrm{~dB}$ limit can be generated. By adding a final-state transfer pulse, our scheme could also be used to generate strong optical squeezing. It is also general enough to be realized in other implementations of parametrically coupled bosonic modes (e.g., superconducting circuits).

Note added: Very recent work by Didier, Qassemi, and Blais analyzes an alternative dissipative squeezing mechanism [43].

\section{ACKNOWLEDGMENTS}

We thank N. Didier and A. Blais for drawing our attention to the possibility of dissipative squeezing, and we acknowledge support from the DARPA ORCHID program through a grant 
from AFOSR, the Emmy-Noether program, the European Research Council, and the ITN cQOM. A.K. thanks A.A.C. for his hospitality at McGill.

\section{APPENDIX A: SEMICLASSICAL PICTURE OF SQUEEZING GENERATION}

As described in the main text, one can obtain a semiclassical understanding of the squeezing generation of our scheme by formally eliminating the cavity from the dynamics; we provide more details on this approach here. In addition to the mechanical resonator quadratures $\hat{X}_{1}$ and $\hat{X}_{2}$, we introduce the cavity quadratures by

$$
\hat{U}_{1}=\left(\hat{d}^{\dagger}+\hat{d}\right) / \sqrt{2} \text { and } \hat{U}_{2}=i\left(\hat{d}^{\dagger}-\hat{d}\right) / \sqrt{2} .
$$

Using the Hamiltonian (2), the Heisenberg-Langevin equations take the form

$$
\begin{aligned}
& \dot{\hat{X}}_{1}=-\left(G_{-}-G_{+}\right) \hat{U}_{2}-\frac{\Gamma_{M}}{2} \hat{X}_{1}+\sqrt{\Gamma_{M}} \hat{X}_{1, \text { in }}, \\
& \dot{\hat{U}}_{2}=\left(G_{-}+G_{+}\right) \hat{X}_{1}-\frac{\kappa}{2} \hat{U}_{2}+\sqrt{\kappa} \hat{U}_{2, \text { in }},
\end{aligned}
$$

and

$$
\begin{aligned}
& \dot{\hat{U}}_{1}=-\left(G_{-}-G_{+}\right) \hat{X}_{2}-\frac{\kappa}{2} \hat{U}_{1}+\sqrt{\kappa} \hat{U}_{1, \text { in }}, \\
& \dot{\hat{X}}_{2}=\left(G_{-}+G_{+}\right) \hat{U}_{1}-\frac{\Gamma_{M}}{2} \hat{X}_{2}+\sqrt{\Gamma_{M}} \hat{X}_{2, \text { in }},
\end{aligned}
$$

where we have also introduced quadratures of the input noise operators $\hat{d}_{\text {in }}$ and $\hat{b}_{\text {in }}$. Note that these are two decoupled sets of equations. They immediately let us understand the backaction-evading limit $G_{+}=G_{-}[16,18]$ : the cavity $\hat{U}_{2}$ quadrature measures the mechanical resonator's quadrature $\hat{X}_{1}$ without disturbing its time evolution. The generation of steady-state squeezing, however, requires that $G_{+}$be slightly smaller than $G_{-}$. In this case, the cavity $\hat{U}_{2}$ quadrature still measures $\hat{X}_{1}$. However, the cavity $\hat{U}_{2}$ quadrature also acts as a force on $\hat{X}_{1}$; we can view this as a weak, coherent feedback force [24] [cf. the first line of Eq. (A1)]. As we now show, this effective feedback leads directly to steady-state squeezing.

It is convenient to work in the Fourier domain, where

$$
f[\omega]=\frac{1}{\sqrt{2 \pi}} \int d t e^{i \omega t} f(t) .
$$

Eliminating the cavity quadratures from the mechanical equations of motion, we find

$$
\begin{aligned}
{\left[-i \omega+\frac{\Gamma_{M}}{2}+i \Sigma[\omega]\right] \hat{X}_{1}[\omega]=} & -\sqrt{\kappa} \frac{i \Sigma[\omega]}{G_{-}+G_{+}} \hat{U}_{2, \text { in }}[\omega] \\
& +\sqrt{\Gamma_{M}} \hat{X}_{1, \text { in }}[\omega]
\end{aligned}
$$

and

$$
\begin{aligned}
{\left[-i \omega+\frac{\Gamma_{M}}{2}+i \Sigma[\omega]\right] \hat{X}_{2}[\omega]=} & \sqrt{\kappa} \frac{i \Sigma[\omega]}{G_{-}-G_{+}} \hat{U}_{1, \text { in }}[\omega] \\
& +\sqrt{\Gamma_{M}} \hat{X}_{2, \text { in }}[\omega],
\end{aligned}
$$

where the self-energy $\Sigma[\omega]=-i\left(G_{-}^{2}-G_{+}^{2}\right) /(\kappa / 2-i \omega)$ is the same for both quadratures. The imaginary part of $\Sigma$ describes damping of the mechanical quadratures by the cavity.
These equations also imply that the correlations $\left\langle\hat{X}_{1} \hat{X}_{2}+\right.$ $\left.\hat{X}_{2} \hat{X}_{1}\right\rangle$ are zero.

The cavity also introduces new noise terms driving each mechanical quadrature. We can parametrize them by an effective temperature in the standard way by considering their magnitude compared to the corresponding cavity-induced damping:

$$
\begin{gathered}
1+2 n_{\mathrm{eff}, X_{1}}[\omega] \equiv \frac{\kappa\left|\Sigma[\omega] /\left(G_{-}+G_{+}\right)\right|^{2}}{-2 \operatorname{Im} \Sigma[\omega]}, \\
1+2 n_{\mathrm{eff}, X_{2}}[\omega] \equiv \frac{\kappa\left|\Sigma[\omega] /\left(G_{-}-G_{+}\right)\right|^{2}}{-2 \operatorname{Im} \Sigma[\omega]} .
\end{gathered}
$$

Taking the low frequency limit, we have

$$
\begin{aligned}
& 1+2 n_{\mathrm{eff}, X_{1}}[0]=\frac{G_{-}-G_{+}}{G_{-}+G_{+}}, \\
& 1+2 n_{\mathrm{eff}, X_{2}}[0]=\frac{G_{-}+G_{+}}{G_{-}-G_{+}} .
\end{aligned}
$$

Thus, while the cavity damps both mechanical quadratures in the same way, the noise added to the $\hat{X}_{1}$ quadrature is much smaller than the noise added to the $\hat{X}_{2}$ quadrature (this is different from coherent parametric driving, where the squeezed $\hat{X}_{1}$ quadrature experiences extra damping whereas $\hat{X}_{2}$ experiences extra negative damping). Moreover, the magnitude of the noise added to the $\hat{X}_{1}$ quadrature is smaller than the the zero-point noise one would associate with the optical damping $\Gamma_{\text {opt }} \equiv-2 \operatorname{Im} \Sigma[0]$, i.e., $n_{\text {eff, } X_{1}}[0]<0$. We thus see that the cavity effectively acts as a squeezed reservoir, i.e., a reservoir whose force noise is quadrature squeezed. If this cavity-induced dissipation dominates the intrinsic mechanical dissipation, this directly yields squeezing of the mechanical resonator.

Finally, it is interesting to note that for a fixed $\mathcal{C} \gg 1$, the optimal ratio of $G_{+} / G_{-}$given in Eq. (5) of the main text can be given a simple interpretation in terms of the effective optical damping $\Gamma_{\text {opt }}=4 \mathcal{G}^{2} / \kappa$ introduced above (with $\mathcal{G}^{2}=$ $G_{-}^{2}-G_{+}^{2}$ ). Using the result of Eq. (5), we have

$$
\begin{aligned}
\left.\Gamma_{\mathrm{opt}}\right|_{\mathrm{optimal}} & \simeq \Gamma_{M}\left(1+2 n_{\mathrm{th}}\right) \sqrt{\frac{4 \mathcal{C}}{1+2 n_{\mathrm{th}}}} \\
& \simeq \Gamma_{M}\left(1+2 n_{\mathrm{th}}\right) e^{2 r} .
\end{aligned}
$$

One can easily confirm that this is exactly the rate at which the $\beta$ mode is heated by the mechanical bath (in the large$r$ limit). We thus see that the optimal coupling condition represents a simple impedance matching: the rate at which the engineered reservoir (the cavity) extracts quanta from the $\beta$ mode should match the rate at which it is "heated" by the intrinsic mechanical dissipation.

\section{APPENDIX B: SQUEEZED-STATE PURITY}

In general, the effective thermal occupancy $n_{\text {eff }}$ (quantifying the purity of the mechanical state) is defined by

$$
4\left\langle\hat{X}_{1}^{2}\right\rangle\left\langle\hat{X}_{2}^{2}\right\rangle=\left(1+2 n_{\mathrm{eff}}\right)^{2}
$$


cf. Eq. (7), since $\left\langle\hat{X}_{1} \hat{X}_{2}+\hat{X}_{2} \hat{X}_{1}\right\rangle=0$. The two variances in terms of the Bogoliubov mode $\hat{\beta}$ read

$$
\begin{aligned}
& 2\left\langle\hat{X}_{1}^{2}\right\rangle=e^{-2 r}\left(1+2\left\langle\hat{\beta}^{\dagger} \hat{\beta}\right\rangle+\langle\hat{\beta} \hat{\beta}\rangle+\left\langle\hat{\beta}^{\dagger} \hat{\beta}^{\dagger}\right\rangle\right), \\
& 2\left\langle\hat{X}_{2}^{2}\right\rangle=e^{2 r}\left(1+2\left\langle\hat{\beta}^{\dagger} \hat{\beta}\right\rangle-\langle\hat{\beta} \hat{\beta}\rangle-\left\langle\hat{\beta}^{\dagger} \hat{\beta}^{\dagger}\right\rangle\right) .
\end{aligned}
$$

As discussed in the main text, optimal squeezing involves a tradeoff between maximizing the squeeze parameter $r$ (which requires large $G_{+} / G_{-}$) and maximizing the effective coupling $\mathcal{G}$ to the Bogoliubov mode (which requires small $G_{+} / G_{-}$). The maximum squeezing at fixed $\mathcal{C}$ thus corresponds to $\beta$ not being in its vacuum state. Equations (B1) and (B2) thus imply that $n_{\text {eff }} \neq 0$; cf. Fig. 2 . The optimally squeezed state is thus in general a thermal squeezed state.

As is also discussed in the main text, there is a general regime where one has large optimized squeezing while at the same time having an almost pure state. This occurs for the "moderately strong coupling" regime where $\mathcal{C} \gg 1$ while at the same time $\mathcal{G}<\kappa$ (no strong-coupling hybridization of $\hat{\beta}$ and the cavity). The latter condition is satisfied for optimized couplings as long as $\mathcal{C}$ is small enough to satisfy

$$
\mathcal{C} \leqslant \frac{1}{16}\left(\frac{\kappa}{\Gamma_{M}}\right)^{2} \frac{1}{2 n_{\mathrm{th}}+1} .
$$

In this regime, one can achieve optimized steady-state squeezing well beyond $3 \mathrm{~dB}$ and a low entropy: $n_{\text {eff }} \sim(\sqrt{2}-1) / 2 \approx$ 0.2 (cf. Fig. 2).

To see this, we now focus on the limit of large cooperativities $\mathcal{C}=4 G_{-}^{2} /\left(\kappa \Gamma_{M}\right)$ while keeping away from the strong-coupling regime. Formally, one can perform this limit by sending $\Gamma_{M} \rightarrow 0$ (in contrast to the previous discussions) while keeping all other parameters fixed. Then, for $\mathcal{G}<\kappa$ and for large $\mathcal{C}$ we find

$$
\left(1+2 n_{\mathrm{eff}}\right)^{2} \approx 2\left(1+\frac{4 G_{-}^{2}\left(1+2 n_{\mathrm{th}}\right)+\kappa^{2} n_{\mathrm{th}}}{\kappa^{2} \sqrt{1+2 n_{\mathrm{th}}}} \frac{1}{\sqrt{C}}\right) \rightarrow 2
$$

or $n_{\text {eff }} \approx(\sqrt{2}-1) / 2$. Thus, independent of the choice of parameters, one always finds that the effective thermal occupancy $n_{\text {eff }} \sim(\sqrt{2}-1) / 2$ in the moderately strong-coupling limit (i.e., $\mathcal{C} \gg 1$ while $\mathcal{G}<\kappa$ ). Note that in this regime, the Bogoliubov mode $\beta$ is characterized by $\left\langle\hat{\beta}^{\dagger} \hat{\beta}\right\rangle,\langle\hat{\beta} \hat{\beta}\rangle \rightarrow 1 / 4$. This follows from the fact that $\langle\hat{\beta} \hat{\beta}\rangle \rightarrow\left\langle\hat{\beta}^{\dagger} \hat{\beta}\right\rangle$ as $\mathcal{C} \rightarrow \infty$ and Eqs. (B1)-(B4).

\section{APPENDIX C: COMPARISON AGAINST MEASUREMENT-BASED SCHEMES}

\section{Comparison against measurement-based feedback squeezing}

As discussed in the main text, the ability of our scheme to generate large amounts of stationary quantum squeezing with low entropy indicates that it outperforms what is possible with a simple coherent parametric driving (i.e., spring constant modulation). Here, we also suggest that it has significant advantages compared to schemes for squeezing based on a backaction-evading (BAE) single-quadrature measurement plus feedback [18]. As our scheme can be viewed as a kind of coherent feedback operation, this apparent advantage is reminiscent of claims made in Ref. [24]. That work also provides specific examples where coherent feedback control schemes can outperform Gaussian measurement-based schemes.

For simplicity, we focus on the regime of large cooperatives $\mathcal{C}=4 G_{-}^{2} /\left(\kappa \Gamma_{M}\right)$ where large squeezing is possible both in our scheme and the BAE measurement scheme. Since the latter scheme was only analyzed in the limit of no strong-coupling effects, we consider the same limit here: we keep $G_{+}, G_{-} \ll$ $\kappa$ while having $\mathcal{C} \rightarrow \infty$ by taking $\Gamma_{M} \rightarrow 0$. If we simply focus on the maximum possible squeezing achievable at a fixed cooperativity, there is no fundamental advantage of our dissipative scheme over the BAE scheme, as both predict a scaling:

$$
2\left\langle\hat{X}_{1}^{2}\right\rangle \approx \sqrt{\frac{1+2 n_{\mathrm{th}}}{\mathcal{C}}}
$$

Of course in practice, achieving this value using BAE measurement and feedback could be very challenging, as it requires near-ideal measurements and feedback.

However, the advantage of our coherent feedback scheme (even on an ideal, fundamental level) becomes apparent when studying the purity of the generated squeezed state. As already discussed, if we stay out of the strong-coupling regime, the mechanical squeezed state is almost in a pure state, with the effective number of thermal quanta $n_{\text {eff }}$ tending to $\sim 0.2$ for large $\mathcal{C}$ as per Eq. (B4). In contrast, the BAE measurementplus-feedback scheme yields

$$
\left(1+2 n_{\text {eff }}\right)^{2}=\sqrt{1+2 n_{\text {th }}} \sqrt{\mathcal{C}} \rightarrow \infty
$$

in the same limit. Thus, our scheme (an example of coherent feedback) yields a far more pure state than the measurementplus-feedback approach. This represents a significant advantage over the measurement-based approach.

We note that one could improve the state purity achieved in the BAE measurement scheme by measuring both quadratures of the cavity output (instead of measuring only the quadrature that contains information on the coupled mechanical quadrature $\hat{X}_{1}$ ). One would thus also learn something about the backaction noise driving the unmeasured quadrature $\hat{X}_{2}$. This would reduce the conditional variance, and thus improve the state purity. The analogous situation involving the dispersive measurement of a qubit is well studied; see, e.g., [44].

\section{Comparison against stroboscopic measurements}

An alternative way of generating mechanical resonator squeezing is to perform a stroboscopic QND position measurement as suggested in Ref. [17]. In this scheme, the measurement rate is modulated in time periodically. The backaction-evading scheme is basically a stroboscopic scheme with a particular choice for how the measurement rate is modulated (i.e., sinusoidally). Instead of the cooperativity, the crucial parameter determining the amount of squeezing generated is now given by the measurement rate. Based on the analysis of Ref. [17], the scaling of squeezing and state purity of the stroboscopic measurement scheme is essentially the same as in the backaction-evading scheme. 


\section{Comparison against pulsed optomechanics schemes}

Let us now compare our dissipative scheme to a pulsed optomechanical scheme, where large-amplitude pulses of light driving an optomechanical cavity are used to realize effective strong position measurements, which, hence, can generate squeezing [22]. We note that this scheme requires $\kappa \gg \Omega$. Since this scheme effectively realizes a strong position measurement, the amount of squeezing scales with the parameter $(\kappa / G)^{2}$, where $G=g_{0} \sqrt{N_{P}}$, and $N_{P}$ is the mean number of photons per light pulse. This is in marked contrast to our dissipative scheme, where the cooperativity $\mathcal{C}$ determines the amount of squeezing. In addition, the pulsed scheme does not generate truly stationary squeezing, which is again in sharp contrast to our dissipative scheme. Finally, we note that using the pulsed scheme, it is currently challenging to get squeezing from this scheme experimentally [45]. However, our scheme should generate large amounts of squeezing even for state-of-the-art experiments; cf. Fig. 3 of our manuscript.

\section{APPENDIX D: EFFECTIVE LINDBLAD MASTER EQUATION}

In this appendix, we derive an effective Lindblad master equation which describes the effects of the cavity (the engineered reservoir) on the mechanical resonator. Such an approach is common in studies of reservoir engineering; in contrast, the approach we use in the main text goes beyond this approximation. We start by considering the Hamiltonian (3)

$$
\hat{H}=-\hbar \mathcal{G} \hat{d}^{\dagger} \hat{\beta}+\text { H.c., }
$$

where $\mathcal{G}^{2}=G_{-}^{2}-G_{+}^{2}, \hat{\beta}=\hat{b} \cosh r+\hat{b}^{\dagger} \sinh r, \cosh r=$ $G_{-} / \mathcal{G}$, and $\sinh r=G_{+} / \mathcal{G}$. Taking the limit of a large cavity damping rate $\kappa$, one can use standard techniques [46] to eliminate the cavity and derive a Lindblad-form master equation for the reduced density matrix of the mechanical resonator $\hat{\rho}$. This takes the form

$$
\dot{\hat{\rho}}=\Gamma_{M}\left(n_{\mathrm{th}}+1\right) \mathcal{D}[\hat{b}] \hat{\rho}+\Gamma_{M} n_{\mathrm{th}} \mathcal{D}\left[\hat{b}^{\dagger}\right] \hat{\rho}+\Gamma_{\mathrm{opt}} \mathcal{D}[\hat{\beta}] \hat{\rho},
$$

where $\mathcal{D}[\hat{A}]=\hat{A} \hat{\rho} \hat{A}^{\dagger}-\hat{A}^{\dagger} \hat{A} \hat{\rho} / 2-\hat{\rho} \hat{A}^{\dagger} \hat{A} / 2$. Expressing the Bogoliubov mode in terms of the original operators $\hat{b}$ and $\hat{b}^{\dagger}$ yields

$$
\begin{aligned}
\dot{\hat{\rho}}= & {\left[\Gamma_{M}\left(n_{\mathrm{th}}+1\right)+\Gamma_{\mathrm{opt}} \cosh ^{2} r\right] \mathcal{D}[\hat{b}] \hat{\rho} } \\
& +\left[\Gamma_{M} n_{\mathrm{th}}+\Gamma_{\mathrm{opt}} \sinh ^{2} r\right] \mathcal{D}\left[\hat{b}^{\dagger}\right] \hat{\rho} \\
& +\Gamma_{\mathrm{opt}} \cosh r \sinh r \mathcal{D}_{S}[\hat{b}] \hat{\rho} \\
& +\Gamma_{\mathrm{opt}} \cosh r \sinh r \mathcal{D}_{S}\left[\hat{b}^{\dagger}\right] \hat{\rho}
\end{aligned}
$$

where $\Gamma_{\text {opt }}=4 \mathcal{G}^{2} / \kappa$ and $\mathcal{D}_{S}[\hat{b}] \hat{\rho}=\hat{b} \hat{\rho} \hat{b}-\hat{b} \hat{b} \hat{\rho} / 2-\hat{\rho} \hat{b} \hat{b} / 2$. The last two terms on the right-hand side (RHS) of this equation do not conserve the number of mechanical quanta, and are directly responsible for the generation of squeezing. This Lindblad master equation is similar to the one discussed in the context of dissipative preparation of spin squeezed atomic ensembles [32].

Using Eq. (D1), we can again calculate the optimal value of $G_{+}$which maximizes the squeezing (with other parameters and $G_{-}$held fixed). We find that this approach leads to the same expression which we have already found using the full theory, Eq. (5) in the main text. For this optimized coupling and in the limit of large cooperativity $\mathcal{C}$, the Lindblad approach predicts

$$
2\left\langle\hat{X}_{1}^{2}\right\rangle \approx \sqrt{\frac{1+2 n_{\mathrm{th}}}{\mathcal{C}}} .
$$

Comparing with Eq. (6) in the main text, we see that the Lindblad approach misses the saturation of squeezing to $\Gamma_{M}\left(1+2 n_{\mathrm{th}}\right) / \kappa$ in the large- $\mathcal{C}$ limit. The approximations used to derive the Lindblad master equation tacitly assume $\kappa \rightarrow \infty$, and thus neglect the finite rate at which the cavity is able to expel energy extracted from the mechanical resonator.

When focusing on the purity of the squeezed state, we find that the Lindblad master equation predicts

$$
\left(1+2 n_{\mathrm{eff}}\right)^{2} \approx 2+\frac{2 n_{\mathrm{th}}}{\sqrt{2 n_{\mathrm{th}}+1}} \frac{1}{\sqrt{C}} .
$$

This is in strong contrast to the prediction $\left(1+2 n_{\text {eff }}\right)^{2} \sim \sqrt{\mathcal{C}}$ of the full theory. This is because the Lindblad master equation cannot capture strong-coupling effects.

\section{APPENDIX E: CAVITY OUTPUT SPECTRA AND SQUEEZING DETECTION}

Let us consider the cavity output spectrum,

$$
S[\omega]=\int \mathrm{d} t e^{i \omega t}\left\langle\delta \hat{a}_{\text {out }}^{\dagger}(t) \delta \hat{a}_{\text {out }}(0)\right\rangle,
$$

where $\delta \hat{a}_{\text {out }}=\hat{a}_{\text {out }}-\left\langle\hat{a}_{\text {out }}\right\rangle$ and $\hat{a}_{\text {out }}+\hat{a}_{\text {in }}=\sqrt{\kappa} \hat{a}$ for an ideal, single-sided cavity [39]. We find that

$$
S\left[\omega-\omega_{\mathrm{cav}}\right]=\frac{16 \kappa \Gamma_{M}\left[G_{+}^{2}\left(n_{\mathrm{th}}+1\right)+G_{-}^{2} n_{\mathrm{th}}\right]}{|N[\omega]|^{2}},
$$

where

$$
N[\omega]=4 \mathcal{G}^{2}+\left(\Gamma_{M}-2 i \omega\right)(\kappa-2 i \omega) .
$$

As shown in Fig. 4, the coupling to the $\beta$ mode gives rise to weight in the output spectrum near the cavity resonance (a)

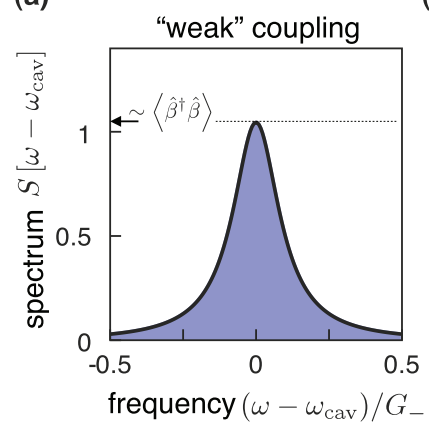

(b)

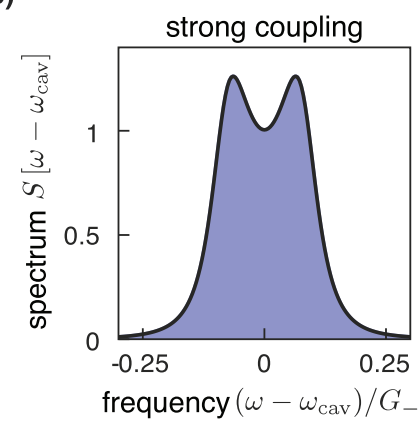

FIG. 4. (Color online) Cavity output spectra. (a) Cavity output spectrum for weak coupling. The spectrum at $\omega-\omega_{\text {cav }}$ as well as the area of the spectrum are directly proportional to the occupancy $\left\langle\hat{\beta}^{\dagger} \hat{\beta}\right\rangle$. (b) If we increase the cooperativity, we observe a normal mode splitting. This is a signature of the "strong-coupling regime," where the Bogoliubov mode and the photons hybridize. [Parameters: (a) $\Gamma_{M} / \kappa=10^{-4}, \quad n_{\text {th }}=10$, and $\mathcal{C}=10^{4}$. (b) Same as (a) but $\mathcal{C}=10^{6}$.] 
frequency. For weak coupling $(\mathcal{G}<\kappa)$, one has a simple Lorentzian peak, whereas for strong coupling $(\mathcal{G}>\kappa)$ a double-peak structure emerges. The condition for this strong coupling to occur was given in Eq. (B3).

As discussed in the main text [cf. Eq. (8)], one can detect the squeezing of the mechanical resonator by first measuring $\left\langle\hat{\beta}^{\dagger} \hat{\beta}\right\rangle$ from the integrated output spectrum, and then using this to bound the variance of the $\hat{X}_{1}$ quadrature. The general expression for the $\hat{X}_{1}$ variance in terms of the Bogoliubov mode $\hat{\beta}$ is given in Eq. (B2). Since in general

$$
|\langle\hat{\beta} \hat{\beta}\rangle| \leqslant\left\langle\hat{\beta}^{\dagger} \hat{\beta}\right\rangle+\frac{1}{2}
$$

(which can be shown by using the Cauchy-Schwarz inequality), one finds a general upper bound for the squeezing,

$$
2\left\langle\hat{X}_{1}^{2}\right\rangle \leqslant 2 e^{-2 r}\left[1+2\left\langle\hat{\beta}^{\dagger} \hat{\beta}\right\rangle\right] .
$$

In the limit of large cooperativity, and hence large $r$, one can also find a lower bound by making use of the theoretical predictions (for $\kappa / \Omega=0$ ). For any value of the squeezing parameter $r$, we find that $\langle\hat{\beta} \hat{\beta}\rangle \sim\left\langle\hat{\beta}^{\dagger} \hat{\beta}\right\rangle$. In the limit of large cooperativities (and hence large $r$ ),

$$
\langle\hat{\beta} \hat{\beta}\rangle \approx\left[1+\left(4 \frac{n_{\text {th }}+1}{2 n_{\text {th }}+1}-2\right) e^{-2 r}\right]\left\langle\hat{\beta}^{\dagger} \hat{\beta}\right\rangle .
$$

Thus,

$$
2\left\langle\hat{X}_{1}^{2}\right\rangle \approx e^{-2 r}\left[1+4 \zeta\left\langle\hat{\beta}^{\dagger} \hat{\beta}\right\rangle\right]
$$

where

$$
\zeta=1+\left(2 \frac{1+n_{\mathrm{th}}}{1+2 n_{\mathrm{th}}}-1\right) e^{-2 r} \geqslant 1 .
$$

Using this estimate, we finally find the lower bound,

$$
2\left\langle\hat{X}_{1}^{2}\right\rangle \geqslant e^{-2 r}\left(1+4\left\langle\hat{\beta}^{\dagger} \hat{\beta}\right\rangle\right) .
$$

\section{APPENDIX F: EFFECTS OF FINITE SIDEBAND PARAMETERS}

To consider effects due to finite sideband parameters, we perturbatively solve the quantum Langevin equations for the cavity and mechanical resonator operators by using the full, time-dependent Hamiltonian (2), keeping the leading corrections in $\kappa / \Omega$. We also compare with a full numerical solution of the equations.

In Fig. 5, the quadrature variance $\left\langle\hat{X}_{1}^{2}\right\rangle$ is shown as a function of the blue laser driving strength $G_{+} / G_{-}$including bad cavity effects. The black curve depicts our analytical perturbative expression (which is too lengthy to be reported here), whereas the orange circles show the result of a numerical simulation of (2). Note that $\left\langle\hat{X}_{1}^{2}\right\rangle$ is strongly nonmonotonic. We find that a unique optimum of the driving strength $G_{+} / G_{-}$ maximizing steady-state squeezing still exists [cf. Fig. 5(b)]. A plot showing the maximized squeezing as a function of the cooperativity $\mathcal{C}$ for fixed sideband parameter $\kappa / \Omega$ and decay rates $\Gamma_{M} / \kappa$ is shown in Fig. 3, where parameters of a state-of-the-art experiment [42] have been assumed. Figure 6 also shows maximized squeezing as a function of $\mathcal{C}$ for experimental parameters of state-of-the-art photonic crystal experiments [47].

Let us now discuss the influence of the counterrotating terms in more detail. For small cooperativities, the effect of
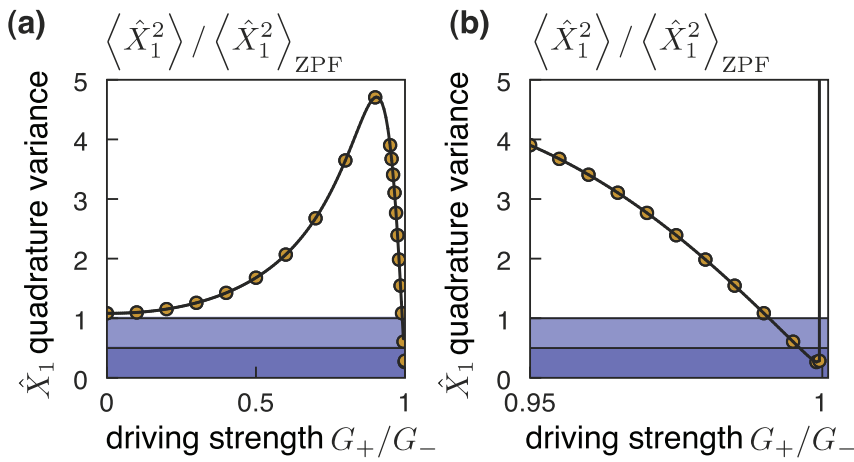

FIG. 5. (Color online) (a) Quadrature variance $\left\langle\hat{X}_{1}^{2}\right\rangle$ as a function of the blue driving strength $G_{+} / G_{-}$including finite sideband parameter effects. The black curve shows the analytical result whereas the circles represent findings due to a numerical simulation of the full Hamiltonian (2). (b) Zoom of (a). We see that the mechanical resonator can be squeezed beyond the $3 \mathrm{~dB}$ limit. (Parameters: $\Gamma_{M} / \kappa=10^{-4}, \kappa / \Omega=1 / 50, n_{\mathrm{th}}=100$, and $\mathcal{C}=5 \times 10^{6}$.)

the counterrotating terms is small. As we increase the cooperativity, the squeezing parameter $r$ also increases [cf. Eq. (5) for the case $\kappa / \Omega=0]$, such that the counterrotating terms become more and more important. Since $r \approx \ln \left[4 \mathcal{C} /\left(1+2 n_{\text {th }}\right)\right] / 4$ for $\kappa / \Omega=0$ and large $\mathcal{C}$, we find that the smaller $n_{\text {th }}$, the earlier these corrections become important. When increasing the cooperativity further, maximum squeezing is assumed first, after which squeezing gets lost again.

\section{APPENDIX G: AVOIDING THE PARAMETRIC INSTABILITY}

It turns out that due to the two-tone driving, the radiation pressure force $F \propto\left|\bar{a}_{+} e^{-i \omega_{+} t}+\bar{a}_{-} e^{-i \omega_{-} t}\right|^{2}$ oscillates at twice the mechanical frequency, since $\omega_{ \pm}=\omega_{\text {cav }} \pm \Omega$. In an

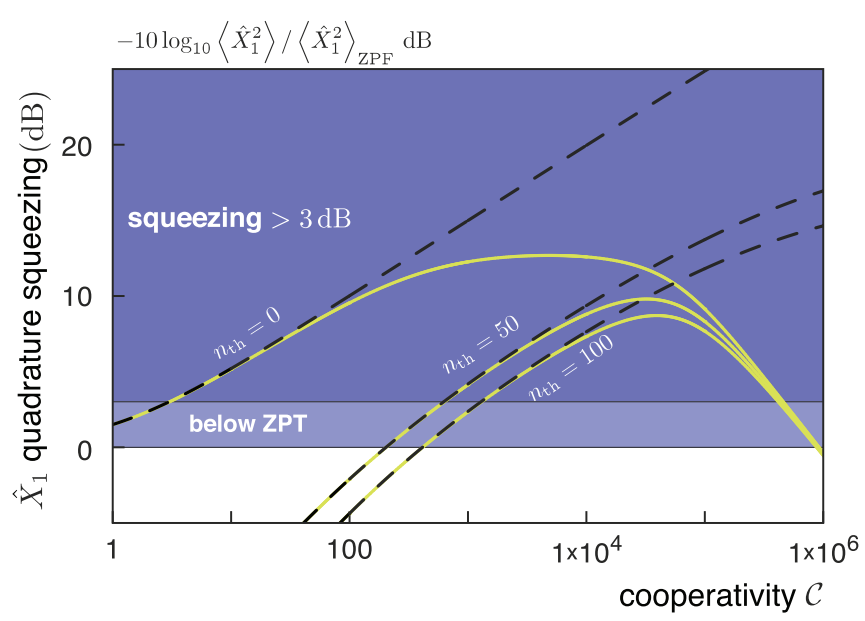

FIG. 6. (Color online) Squeezing as a function of the cooperativity for optimized driving strengths where realistic experimental parameters are assumed [47]. The yellow line and the white dashed lines show the theory including effects due to a finite sideband parameter. The black dashed line shows the expectation for $\kappa / \Omega=$ 0 . Thus, squeezing beyond $3 \mathrm{~dB}$ is expected for state-of-the-art experiments. (Parameters: $\Gamma_{M} / \kappa=10^{-4}, \kappa / \Omega=1 / 10$.) 


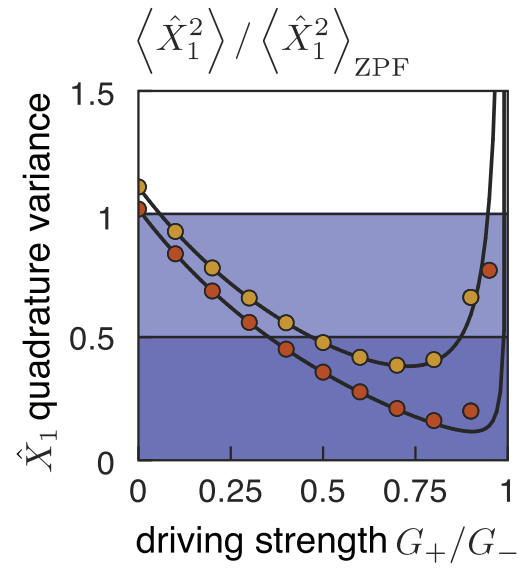

FIG. 7. (Color online) Quadrature variance $\left\langle\hat{X}_{1}^{2}\right\rangle$ as a function of the blue driving strength $G_{+} / G_{-}$including finite sideband parameter effects and effects of a third tone; cf. Eq. (G1). The black curve $\left\langle\hat{X}_{1}^{2}\right\rangle$ includes the effects of finite sideband parameters but ignores the third tone, i.e., $G_{3}=0$. The circles represent results of a numerical simulation of the full Hamiltonian including finite sideband effects and effects due to the third tone. [Parameters: $\Gamma_{M} / \kappa=10^{-4}, \kappa / \Omega=$ $1 / 50, n_{\text {th }}=50$, and $\mathcal{C}=10^{3}$ (upper curve) and $\mathcal{C}=10^{4}$ (lower curve), respectively.]

experiment, these oscillations can yield parametric instabilities if the mechanical resonator frequency $\Omega$ is strongly temperature-dependent [37]. To suppress this instability, one can add a third driving tone to cancel the oscillations of the radiation pressure force at $2 \Omega$ [38]. In the following, we show that the influence of this third driving tone on the generation of steady-state squeezing is small for typical experimental parameters.

The third driving tone can be included in our theory by adding the driving term

$$
\hat{H}_{\text {drive,add }}=\hbar \alpha_{3}\left(e^{-i \omega_{3} t+i \varphi} \hat{a}^{\dagger}+\text { H.c. }\right)
$$

to the Hamiltonian (1). One then finds that the radiation pressure force reads

$$
F \propto\left|\bar{a}_{+} e^{-i \omega_{+} t}+\bar{a}_{-} e^{-i \omega_{-} t}+\bar{a}_{3} e^{-i \omega_{3} t+i \varphi}\right|^{2} .
$$

When choosing $\omega_{3}=\omega_{\text {cav }}-3 \Omega$ [38] we find that the component of $F$ oscillating at $2 \Omega$ vanishes if $\varphi=\pi$ and $\bar{a}_{3}=\bar{a}_{+}$. Linearizing the resulting Hamiltonian again and going into an interaction picture with respect to the free cavity and mechanical resonator Hamiltonian, we find that the third drive tone gives rise to an additional term,

$$
\hat{H}_{\text {new }}=\hbar G_{3} e^{3 i \Omega t}\left[\hat{b} e^{-i \Omega t}+\hat{b}^{\dagger} e^{i \Omega t}\right] \hat{d}^{\dagger}+\text { H.c. },
$$

to the linearized Hamiltonian (2), where $G_{3}=g_{0} \bar{a}_{3}=G_{+}$.

The impact of this additional counterrotating term on the generation of steady-state squeezing is shown in Fig. 7. In this figure, the quadrature variance $\left\langle\hat{X}_{1}^{2}\right\rangle$ is shown as a function of the blue laser driving strength $G_{+} / G_{-}$including all counterrotating terms. For medium cooperativity, the influence of the third tone is negligible. For larger cooperativity, deviations become visible as $G_{+} \rightarrow G_{-}$. However, the minimum value of $\left\langle\hat{X}_{1}^{2}\right\rangle$ is changed little and lies still well beyond $3 \mathrm{~dB}$.

Let us now briefly discuss why the deviations become apparent as we increase the cooperativity and as we approach $G_{+} \rightarrow G_{-}$. An increase of the cooperativity $\mathcal{C}$ leads to an increase of the squeezing parameter $r$, such that the influence of counterrotating terms becomes larger; cf. the discussion in the previous section. Thus, the influence of the additional, counterrotating terms (G1) increases with increasing $\mathcal{C}$. Additionally, to cancel the unwanted frequency component $2 \Omega$ of the radiation pressure force, we have to choose $G_{3}=G_{+}$. Thus, as we increase $G_{+}$, the magnitude of the additional Hamiltonian (G1) also increases, leading to a larger perturbation of the steady-state quadrature variance.

Thus, to conclude, we can avoid the parametric instability by adding a third driving tone while still generating squeezing well beyond $3 \mathrm{~dB}$.
[1] We use the standard quantum optics definition, where "nonclassical" describes any state that cannot be described by a nonsingular $P$ function (see, e.g., [2]).

[2] C. C. Gerry and P. L. Knight, Introductory Quantum Optics (Cambridge University Press, Cambridge, 2005).

[3] C. M. Caves, K. S. Thorne, R. W. P. Drever, V. D. Sandberg, and M. Zimmermann, Rev. Mod. Phys. 52, 341 (1980).

[4] S. L. Braunstein and P. v. Loock, Rev. Mod. Phys. 77, 513 (2005).

[5] D. F. Walls and G. J. Milburn, Quantum Optics, 2nd ed. (Springer, Berlin, 2008).

[6] G. Milburn and D. Walls, Opt. Commun. 39, 401 (1981).

[7] T. J. Kippenberg and K. J. Vahala, Science 321, 1172 (2008).

[8] F. Marquardt and S. M. Girvin, Physics 2, 40 (2009).

[9] P. Meystre, Ann. Phys. 525, 215 (2013).

[10] M. Aspelmeyer, T. J. Kippenberg, and F. Marquardt, arXiv:1303.0733.

[11] A. Mari and J. Eisert, Phys. Rev. Lett. 103, 213603 (2009).

[12] M. J. Woolley, A. C. Doherty, G. J. Milburn, and K. C. Schwab, Phys. Rev. A 78, 062303 (2008).
[13] A. Nunnenkamp, K. Børkje, J. G. E. Harris, and S. M. Girvin, Phys. Rev. A 82, 021806 (2010).

[14] J.-Q. Liao and C. K. Law, Phys. Rev. A 83, 033820 (2011).

[15] M. Schmidt, M. Ludwig, and F. Marquardt, New J. Phys. 14, 125005 (2012).

[16] V. B. Braginsky, Y. I. Vorontsov, and K. S. Thorne, Science 209, 547 (1980).

[17] R. Ruskov, K. Schwab, and A. N. Korotkov, Phys. Rev. B 71, 235407 (2005).

[18] A. A. Clerk, F. Marquardt, and K. Jacobs, New J. Phys. 10, 095010 (2008).

[19] A. Szorkovszky, A. C. Doherty, G. I. Harris, and W. P. Bowen, Phys. Rev. Lett. 107, 213603 (2011).

[20] A. Szorkovszky, G. A. Brawley, A. C. Doherty, and W. P. Bowen, Phys. Rev. Lett. 110, 184301 (2013).

[21] K. Jähne, C. Genes, K. Hammerer, M. Wallquist, E. S. Polzik, and P. Zoller, Phys. Rev. A 79, 063819 (2009).

[22] M. R. Vanner, I. Pikovski, G. D. Cole, M. S. Kim, C. Brukner, K. Hammerer, G. J. Milburn, and M. Aspelmeyer, Proc. Natl. Acad. Sci. 108, 16182 (2011). 
[23] T. Botter, D. W. C. Brooks, N. Brahms, S. Schreppler, and D. M. Stamper-Kurn, Phys. Rev. A 85, 013812 (2012).

[24] R. Hamerly and H. Mabuchi, Phys. Rev. Lett. 109, 173602 (2012).

[25] J. Kerckhoff, R. W. Andrews, H. S. Ku, W. F. Kindel, K. Cicak, R. W. Simmonds, and K. W. Lehnert, Phys. Rev. X 3, 021013 (2013).

[26] J. F. Poyatos, J. I. Cirac, and P. Zoller, Phys. Rev. Lett. 77, 4728 (1996).

[27] N. Bergeal, R. Vijay, V. E. Manucharyan, I. Siddiqi, R. J. Schoelkopf, S. M. Girvin, and M. H. Devoret, Nat. Phys. 6, 296 (2010).

[28] B. Peropadre, D. Zueco, F. Wulschner, F. Deppe, A. Marx, R. Gross, and J. J. Garcia-Ripoll, Phys. Rev. B 87, 134504 (2013).

[29] J. I. Cirac, A. S. Parkins, R. Blatt, and P. Zoller, Phys. Rev. Lett. 70, 556 (1993).

[30] P. Rabl, A. Shnirman, and P. Zoller, Phys. Rev. B 70, 205304 (2004).

[31] A. S. Parkins, E. Solano, and J. I. Cirac, Phys. Rev. Lett. 96, 053602 (2006).

[32] E. G. Dalla Torre, J. Otterbach, E. Demler, V. Vuletic, and M. D. Lukin, Phys. Rev. Lett. 110, 120402 (2013).

[33] Y.-D. Wang and A. A. Clerk, Phys. Rev. Lett. 110, 253601 (2013).

[34] H. Tan, G. Li, and P. Meystre, Phys. Rev. A 87, 033829 (2013).

[35] A. Tomadin, S. Diehl, M. D. Lukin, P. Rabl, and P. Zoller, Phys. Rev. A 86, 033821 (2012).
[36] C. K. Law, Phys. Rev. A 51, 2537 (1995).

[37] J. Suh, M. D. Shaw, H. G. LeDuc, A. J. Weinstein, and K. C. Schwab, Nano Lett. 12, 6260 (2012).

[38] S. K. Steinke, K. C. Schwab, and P. Meystre, Phys. Rev. A 88, 023838 (2013).

[39] A. A. Clerk, M. H. Devoret, S. M. Girvin, F. Marquardt, and R. J. Schoelkopf, Rev. Mod. Phys. 82, 1155 (2010).

[40] F. Marquardt, J. P. Chen, A. A. Clerk, and S. M. Girvin, Phys. Rev. Lett. 99, 093902 (2007).

[41] I. Wilson-Rae, N. Nooshi, W. Zwerger, and T. J. Kippenberg, Phys. Rev. Lett. 99, 093901 (2007).

[42] J. D. Teufel, T. Donner, D. Li, J. W. Harlow, M. S. Allman, K. Cicak, A. J. Sirois, J. D. Whittaker, K. W. Lehnert, and R. W. Simmonds, Nature (London) 475, 359 (2011).

[43] N. Didier, F. Qassemi, and A. Blais, arXiv:1307.5311 [Phys. Rev. A (to be published)].

[44] M. Hatridge, S. Shankar, M. Mirrahimi, F. Schackert, K. Geerlings, T. Brecht, K. M. Sliwa, B. Abdo, L. Frunzio, S. M. Girvin, R. J. Schoelkopf, and M. Devoret, Science 339, 178 (2013).

[45] M. R. Vanner, J. Hofer, G. D. Cole, and M. Aspelmeyer, Nat. Commun. 4, 2295 (2013).

[46] C. W. Gardiner and P. Zoller, Quantum Noise (Springer, Berlin, 2000).

[47] J. Chan, T. P. M. Alegre, A. H. Safavi-Naeini, J. T. Hill, A. Krause, S. Groblacher, M. Aspelmeyer, and O. Painter, Nature (London) 478, 89 (2011). 